\title{
High-resolution microspectrometer with an aberration-correcting planar grating
}

\author{
Semen Grabarnik, ${ }^{*}$ Arvin Emadi, Huaiwen Wu, Ger de Graaf, \\ and Reinoud F. Wolffenbuttel \\ Faculty Electrical Engineering, Mathematics and Computer Science, \\ Department of MicroElectronics/Electronic Instrumentation, \\ Delft University of Technology, Mekelweg 4, 2628 CD, \\ Delft, The Netherlands \\ ${ }^{*}$ Corresponding author: s.grabarnik@tudelft.nl
}

Received 13 June 2008; revised 29 September 2008; accepted 21 October 2008; posted 22 October 2008 (Doc. ID 97397); published 27 November 2008

\begin{abstract}
A concept for a highly miniaturized spectrometer featuring a two-component design is presented. The first component is a planar chip that integrates an input slit and aberration-correcting diffraction grating with an image sensor and is fabricated using microelectromechanical systems (MEMS) technologies. Due to the fabrication in a simple MEMS batch process the essential elements of the spectrometer are automatically aligned, and a low fabrication cost per device can be achieved. The second component is a spherical mirror, which is the only external part. The optimized grating structure compensates for aberrations within the spectrometer operating range, resulting in a diffraction-limited performance of the spectrometer optics. The prototype of the device has been fabricated and characterized. It takes a volume of $0.5 \mathrm{~cm}^{3}$ and provides a FWHM spectral resolution of $0.7 \mathrm{~nm}$ over a $350 \mathrm{~nm}$ bandwidth from $420 \mathrm{~nm}$ to $770 \mathrm{~nm}$ combined with an etendue of $7.4 \times 10^{-5} \mathrm{~mm}^{2} \mathrm{sr}$. (c) 2008 Optical Society of America

OCIS codes: $\quad 300.6190,050.1950,220.2740,220.1000$.
\end{abstract}

\section{Introduction}

Compact optical spectrometers are of huge interest in many areas, including industrial, biomedical, space, and scientific applications. Portability of the system is especially important in space applications, where small size and weight are decisive. The spectrometer is often a core part of a versatile instrument and significantly affects the costs of the complete system. If a spectrometer can be fabricated with an integrated circuit-like batch process suited for high volume production, a low unit price would be possible. Micro-optical electromechanical systems (MOEMS) technologies are very suitable for achieving that objective.

The progress in integrated optics and microelectromechanical systems (MEMS) technologies in primarily silicon has brought possibilities for the design of

0003-6935/08/346442-06\$15.00/0

(C) 2008 Optical Society of America compact spectrometers using very small integrated optical and optomechanical components [1]. For example, a $5 \mathrm{~mm} \times 5 \mathrm{~mm}$ MEMS chip with movable micromechanical parts comprising a variable depth lamellar grating was used in a Fourier transform spectrometer [2]. A $1.6 \mathrm{~nm}$ FWHM resolution at a wavelength of $400 \mathrm{~nm}$ and a $5.5 \mathrm{~nm}$ resolution at $800 \mathrm{~nm}$ have been reported. Electron-beam lithography was used in [3] for the fabrication of an imaging diffraction grating employed in a spectrometer as small as $1 \mathrm{~cm}^{3}$. The reported spectroscopic device demonstrated a $5 \mathrm{~nm}$ resolution within a $100 \mathrm{~nm}$ range. Two imaging gratings have been integrated in a planar design, which has resulted in a $45 \mathrm{~mm}^{3}$ microspectrometer with a $3 \mathrm{~nm}$ FWHM resolution over a $300 \mathrm{~nm}$ operating range in the visible region [4]. A spectrometer employing planar waveguide optics and micro-optical parts has been described in [5]. The authors expect a theoretically feasible resolution of $2 \mathrm{~nm}$ within a $300 \mathrm{~nm}$ operating bandwidth based 
on numerical simulations. A 16-channel Fabry-Perot filter array integrated with photosensors and readout electronics has been demonstrated in [6]. In [7] this approach has been extended to a 128-channel Fabry-Perot filter with FWHM spectral resolution varying from $1.7 \mathrm{~nm}$ to $3.8 \mathrm{~nm}$ from channel to channel in the operating range from $722 \mathrm{~nm}$ to $880 \mathrm{~nm}$.

In general, the additional benefits of using MEMS technologies, such as miniaturization of the device and integration of optics with electronic circuits, are at the expense of optical performance [1], and a subnanometer spectral resolution in a wide wavelength range has not been achieved in a compact system to the best of our knowledge. MEMS technologies are inherently planar in the sense that the outof-plane dimension cannot be controlled to any value by design. Controlled anisotropic [8] or isotropic [9] etching of silicon can be used to fabricate integrated nonplanar optical components in silicon. However, the technologies mentioned are suitable for the fabrication of lenses or mirrors, which are smaller than several hundreds of micrometers in diameter and cannot be applied for production of optical elements with a small $F$-number (less than 4 ) and several millimeters in size. The optical system of a MEMSbased spectrometer is, therefore, either restricted to planar optical surfaces only [4] or external nonplanar components should be used [ $\overline{5}]$. It was demonstrated theoretically that a subnanometer spectral resolution cannot be achieved in a compact system using flat components only with a single diffraction grating, due to large optical aberrations [10]. Spherical or aspherical parts are necessary to achieve the low-aberration optical performance of the system required for high spectral resolution.

This article reports on a two-component miniature spectrometer with an entrance slit, diffraction grating, and image sensor integrated on a planar MEMS chip, which is combined with a low-cost commercially available spherical mirror, a single external component. The goal in the design of such a spectrometer is twofold: first, to maximize spectral resolution under dimensional constraints; second, to make a design that is suitable for component fabrication in a simple MEMS batch process, while allowing relatively large tolerances in component alignment during system assembly. The use of a single spherical mirror defines the optical design as a modified Fastie-Ebert mounting [11] (Fig. 1). The FastieEbert configuration is characterized by the grating tilted relative to photosensor to compensate for optical aberrations. Such a design is employed in commercially available spectrometers, for example, in a miniature Hamamatsu spectrograph with spectral resolution of $8-9 \mathrm{~nm} \mathrm{[12]}$. However, unlike these classical Fastie-Ebert mountings, the planarity of the MEMS chip presented here implies that the entrance slit, the grating, and the image sensor are in the same plane. This planar configuration results in poorly compensated aberrations in the case of a linear grating. Aberrations of the optical system can be

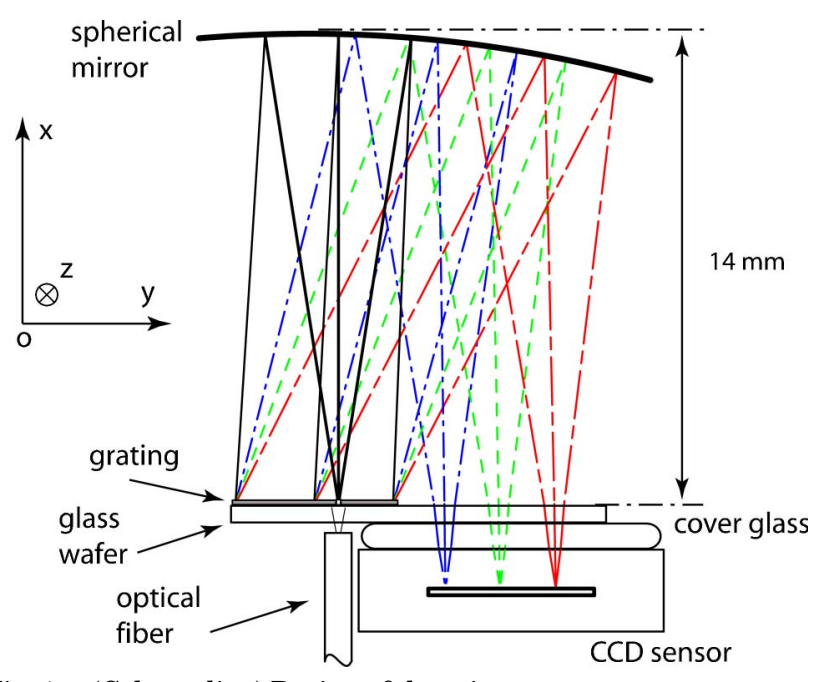

Fig. 1. (Color online) Design of the microspectrometer prototype. A piece of glass wafer integrating a diffraction grating and a transmission slit is mounted on top of the CCD sensor coverglass. An optical fiber is used to illuminate the entrance slit, and the dispersed spectrum is projected onto a CCD chip. Plane oyz of the coordinate system oxyz lies in the grating plane, and the oy axis coincides with the direction of the dispersion.

significantly reduced by an appropriately designed nonlinear grating. Such a grating with slightly curved and not equally spaced grooves is able to provide nearly diffraction-limited performance of the spectrometer imaging system. The proposed design concept involves integration of all spectrometer components on a planar chip, except a single external spherical mirror, combined with an aberrationcorrecting grating and is new to the best of our knowledge. Integration on a planar chip allows the exploitation of all the benefits provided by MEMS technologies. Due to the MEMS processing all of the spectrometer components, with the exception of the spherical mirror, are automatically assembled, and low cost per device in a high-volume production can be achieved. Another potential benefit of the proposed microspectrometer is the possibility to integrate the spectrometer chip with microsensors, such as microfluidic devices, which can be very useful in lab-on-a-chip applications.

\section{Design of the Two-Component Microspectrometer}

The concept described has been implemented in the spectrometer prototype shown schematically in Fig. 1. It consists of a glass plate mounted on top of a commercial CCD sensor. The lithographically fabricated metal pattern on the glass plate integrates diffraction grating and transmission slit, which is illuminated using an optical fiber. The spherical mirror collimates light coming through the entrance slit and enables direction of the beam to the grating. The light diffracted in the first order is focused by the same mirror on the surface of the CCD chip. The design parameters of the spectrometer are pitch of the diffraction grating, focal distance of the spherical mirror, input numerical 
aperture (NA), operating spectral range, CCD pixel size, and dimensions of the CCD sensor.

The grating pitch should be minimized to maximize dispersion (and consequently spectral resolution), but it cannot be made smaller than twice the smallest feature size that can be fabricated using the available lithographic system. In the process used the smallest feature size was about $0.7-0.8 \mu \mathrm{m}$, which set the grating period to $1.6 \mu \mathrm{m}$.

The focal distance of the spherical mirror defines the largest dimension of the spectrometer. One can obtain the following formula using the grating equation:

$$
F=\frac{p d \times \cos (\beta)}{\delta \lambda} .
$$

In Eq. (1) $d$ is the grating period, $p$ is the detector pixel width over which a spectral bandwidth of $\delta \lambda$ is projected, and $\beta$ is the angle of diffraction. According to the Nyquist sampling theorem, $\delta \lambda$ should be smaller than $0.5 \mathrm{~nm}$ to achieve a subnanometer resolution.

The Sony ICX278AL CCD sensor with $4.75 \mu \mathrm{m} \times$ $5.55 \mu \mathrm{m}$ pixels has been selected for the spectrometer prototype because of the relatively small pixel size and high sensitivity, its low price, and the commercial availability of standard compatible readout electronics. Note that current technology allows fabrication of image sensors with smaller pixels of about $2-3 \mu \mathrm{m}$. From Eq. (1), taking into account dimensional constraints and a $4.75 \mu \mathrm{m}$ pixel width, a focal distance results:

$$
F \approx \frac{4.75 \times 10^{-3} \times 1.6 \times 10^{-3} \times 1}{5 \times 10^{-10}}=15 \mathrm{~mm} .
$$

The input numerical aperture (NA) of the spectrometer defines the throughput (or etendue) of the device and should be maximized to increase sensitivity. However, since it is a common practice to provide photonic devices with an optical fiber input, it is useless to make the NA larger than the NA of a multimode fiber (typically 0.22 ). Moreover, the NA of the spectrometer together with the mirror focal distance $F$ defines the size of the grating, $D$, as $D=2 F \times \mathrm{NA}$, and a NA of 0.22 results in a $6.6 \mathrm{~mm}$ grating. A large grating implies a large chip area. The maximum area of a single chip is limited by various technological aspects and also by cost, and that is why compromise is inevitable. Finally, the NA value has been selected to be equal to 0.14 , which results in a $4.2 \mathrm{~mm}$ grating.

Due to the very compact size of the spectrometer, the dimensions of the CCD sensor must be taken into account in the design. These parameters include chip area, size of the chip housing, thickness of the sensor coverglass, and distance from the coverglass to the surface of the CCD silicon chip. The total number of pixels along the horizontal direction defined the operating bandwidth of the spectrometer to be about $350 \mathrm{~nm}$. Subsequently the operating range has been selected to be from $\lambda_{\min }=400 \mathrm{~nm}$ to $\lambda_{\max }=750 \mathrm{~nm}$. The width of the $6 \times 200 \mu \mathrm{m}^{2}$ entrance slit is slightly larger than the diffraction-limited value defined by NA of the spectrometer as $d=\lambda_{\max } / \mathrm{NA}$. With the selected design parameters the etendue of the spectrometer can be calculated as $G=\pi \times l_{\text {slit }} \times w_{\text {slit }} \times$ $\mathrm{NA}^{2}=7.4 \times 10^{-5} \mathrm{~mm}^{2} \mathrm{sr}$.

For the optical design a ray tracing software, ZEMAX [13], has been used. The starting point in the design process was a modified "W"-type Fastie-Ebert configuration in which a diffraction grating with straight grooves was parallel to the image sensor. Due to the parallel position of the grating the system symmetry was distorted and the optical performance was degraded by various optical aberrations. Moving the entrance slit as close to the CCD imager as is possible within the sensor package transforms the spectrometer configuration into a Littrow arrangement and reduces spherical aberration and astigmatism. Subsequently, the structure of the grating grooves has been optimized to compensate for the remaining aberrations (mainly coma) as a next step. A diffraction grating is represented in ZEMAX by a set of grating coefficients $a_{i j}$, which forms a function describing a phase added to the diffracted ray as $P(y, z)=m \Sigma a_{i j} y^{i} z^{j}$, where $m$ is the diffraction order and $y$ and $z^{i j}$ are the coordinates in the grating plane. The direction of the diffracted ray can be found using the local grating periods $d_{y}=2 \pi(\partial P / \partial y)^{-1}$ and $d_{z}=$ $2 \pi(\partial P / \partial z)^{-1}$ for the $o y$ and $o z$ axis, respectively [14]. The optimization functionality of ZEMAX was used to find a set of grating coefficients $a_{i j}$ that provides the smallest possible size of the spectrometer entrance slit image at the wavelengths from the spectrometer operating range in the oy direction, which is the direction of the dispersion in the image plane. Simultaneous optimization of the mirror position and grating coefficients completed the design process. The largest dimension of the device is $14 \mathrm{~mm}$ (Fig. 1), and the size of the glass chip is about $4 \times 9 \mathrm{~mm}^{2}$. The width of the entrance slit image produced by the spectrometer optics when the slit is illuminated with monochromatic light is about $8 \mu \mathrm{m}$ according to numerical simulations. This means that the spectral resolution is limited mainly by the CCD pixel pitch.

\section{Fabrication and Characterization of the Spectrometer}

The chips integrating the diffraction grating and the entrance slit have been fabricated on a glass wafer using a simple two-mask process. Optimized grating coefficients were used to calculate the shape of the grating grooves. Subsequently, the grooves were included in the layout file used in the lithography mask fabrication. The fabrication process started with the deposition of $650 \mathrm{~nm}$ of aluminum on the glass wafer. The first mask was applied to define grating structures, and the gratings were plasma etched to yield $120 \mathrm{~nm}$ deep grooves in the aluminum. The depth of the grooves was controlled by etching time, and the variation in depth over the grating is less than $1 \%$. 


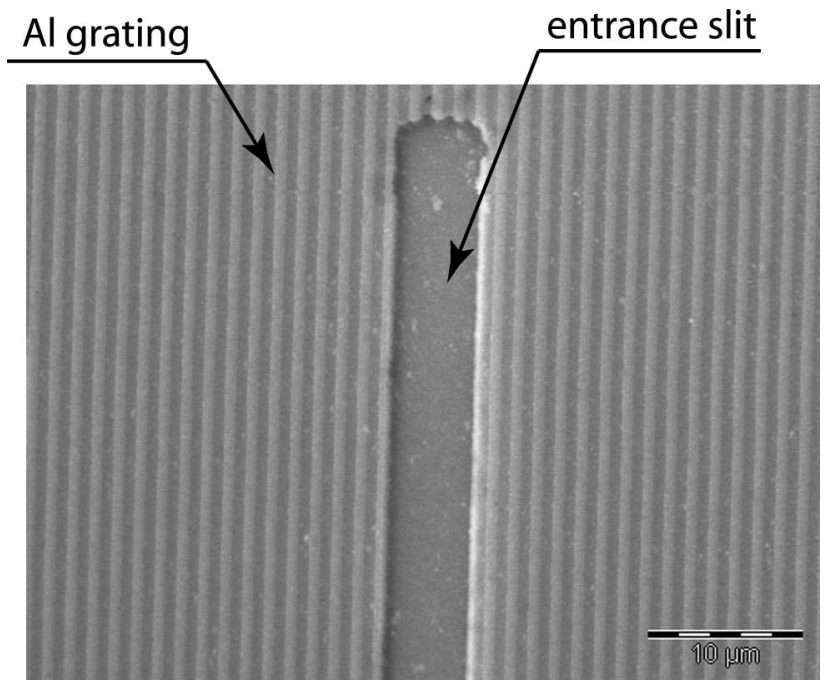

Fig. 2. Scanning electron microscope photograph of a $6 \mu \mathrm{m}$ transmission entrance slit etched in the aluminum diffraction grating.

Finally, using the second mask, the aluminum was patterned and etched to define the transparent input slit and the grating contour. The grooves defined in the first step were protected during the etching. Figure 2 shows a scanning electron microscope photograph of the transparent slit etched in the aluminum grating. The processed wafer was diced and the glass chip was glued on top of the CCD sensor as shown in Fig. 3. The efficiency of the fabricated grating was measured using a He-Ne laser to be $31 \%$ in the first order (at $632 \mathrm{~nm}$ wavelength). The device was aligned with a spherical mirror and the electronics of a Videology 21K137 camera [15] was employed for signal readout.

The performance of the spectrometer was characterized experimentally with a Ne lamp as a test light source. A cleaved tip of a multimode optical fiber was exposed to the Ne lamp, and the second fiber tip was aligned to illuminate the entrance slit of the spectrometer. The resulting spectral pattern was captured by the CCD sensor and observed on the computer screen. The diffraction efficiency of the $\mathrm{Al}$ grating is polarization dependent. However, this dependency has no practical effect on the spectrometer performance, since the light trajectory in the optical system of the spectrometer does not depend on polarization. Moreover, the multimode fiber mixes waveguide modes of different polarizations, providing an unpolarized light at the spectrometer input.

The spectrum of a Ne lamp contains a set of monochromatic lines. Each spectral line illuminates a maximum of two rows of adjacent CCD pixels. The tolerance for the distance between mirror and CCD sensor (direction ox in Fig. 1) has been determined to be about $\pm 20 \mu \mathrm{m}$. When the mirror position is within the tolerance bounds, two rows of pixels are illuminated by a spectral line, and the resolution of the spectrometer is better than $1 \mathrm{~nm}$. If the mirror displacement is larger than the specified tolerance, more than two pixel rows are illuminated, which im- plies degradation of the resolution by more than $30 \%$ as compared to the best possible value. The movement of the mirror parallel to the image plane (direction oy in Fig. 1) results in a shift of the spectral range projected on the CCD sensor, without the significant loss of spectral resolution. Quantitatively, a $100 \mu \mathrm{m}$ displacement of the mirror results in a $20 \mathrm{~nm}$ spectral shift. The adjustment of the mirror position can be used to select a $350 \mathrm{~nm}$ operating bandwidth in the range from $400 \mathrm{~nm}$ to $850 \mathrm{~nm}$.

Figure 4(a) presents the spectral pattern of Ne captured with the CCD camera and a graphical representation of the $\mathrm{Ne}$ spectrum in the range from $420 \mathrm{~nm}$ to $470 \mathrm{~nm}$. The magnified part of the spectrum is shown in Fig. 4(b). The FWHM resolution of the spectrometer can be estimated from the width of a single spectral line in Fig. $\underline{4}$ to be approximately $0.7 \mathrm{~nm}$.

Several peaks are marked with the corresponding measured wavelength values in Fig. 4(b) to identify them with the $\mathrm{Ne}$ spectral lines. The numbers in brackets are reference values from [16]. The positions of spectral lines registered by the spectrometer can be defined with the precision of about $0.3-0.4 \mathrm{~nm}$. This precision is limited by the width of the CCD pixel. The level of stray light is lower than $0.2 \%$, which allows registration of very weak spectral peaks.

\section{Conclusions}

In conclusion, a highly compact spectrometer providing a $0.7 \mathrm{~nm}$ spectral resolution over a $350 \mathrm{~nm}$ range has been designed, fabricated, and characterized. The largest dimension of the optical part is $14 \mathrm{~mm}$,

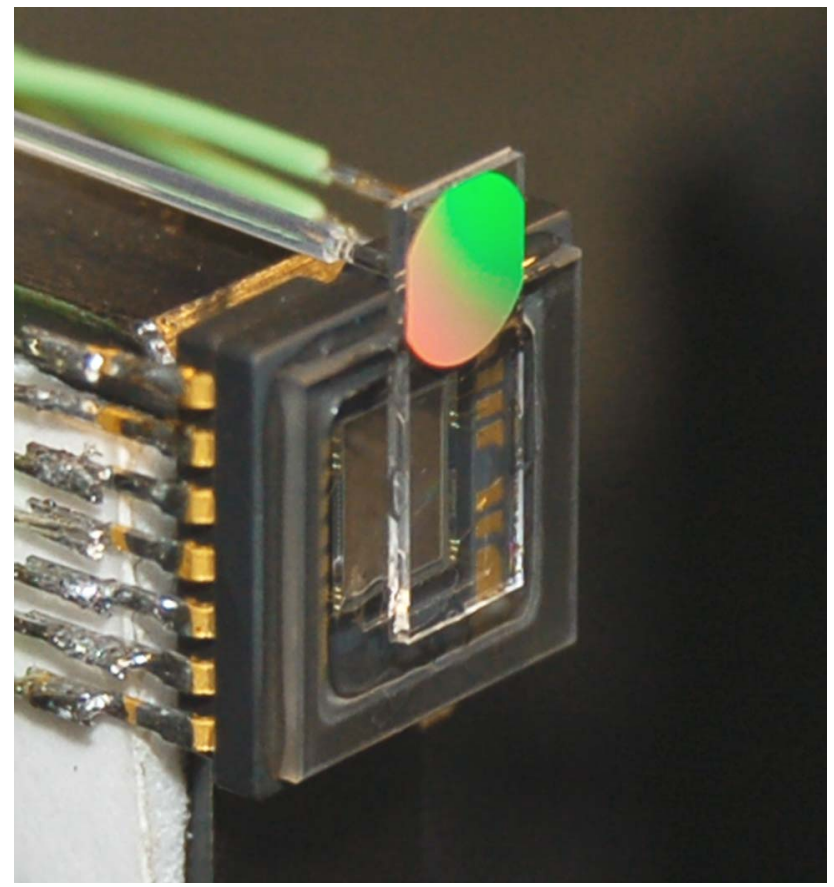

Fig. 3. (Color online) Glass chip glued onto the CCD sensor coverglass. The optical fiber visible in the top left corner of the photo is used to illuminate the transparent entrance slit etched in the diffraction grating. 
(a)

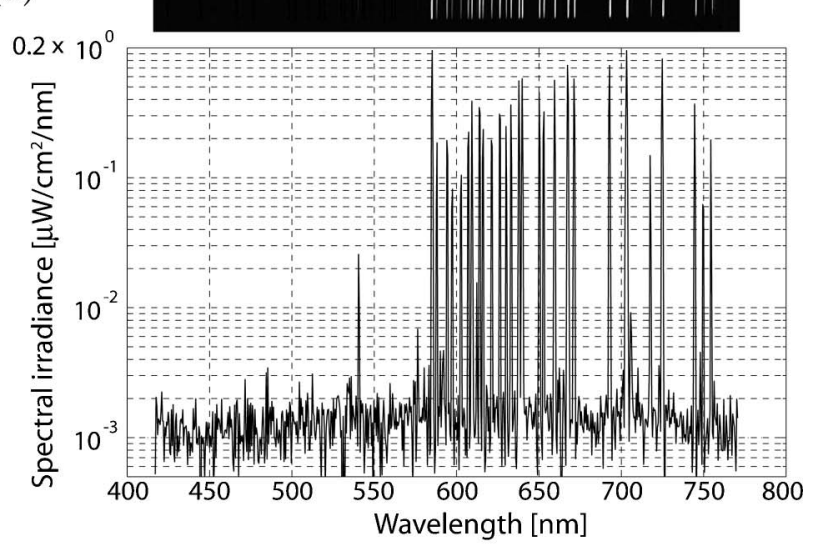

(b)

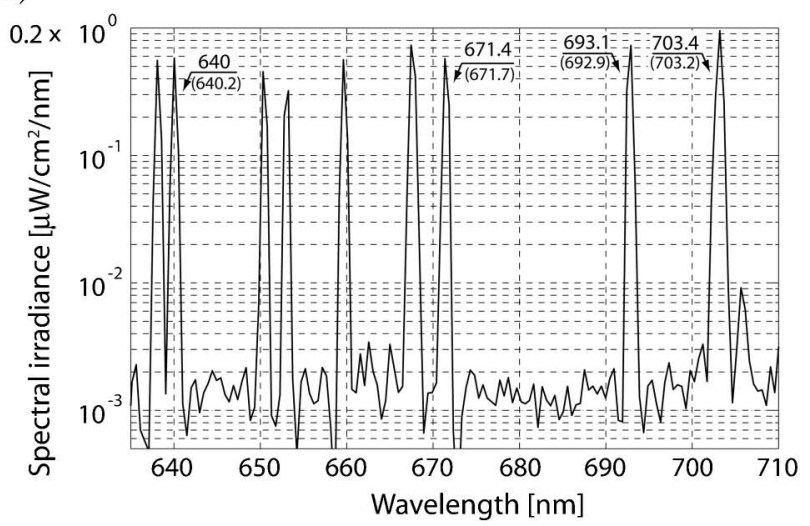

Fig. 4. Spectral pattern captured with the CCD camera and graphical representation of the Ne spectrum in the $350 \mathrm{~nm}$ operating bandwidth of the spectrometer (a) and magnified part of the $\mathrm{Ne}$ spectrum (b). Some of the spectral lines are marked with the corresponding measured peak wavelength values, while the numbers in brackets are reference values.

and the volume is about $0.5 \mathrm{~cm}^{3}$. Such a small volume is combined with a performance comparable to that of conventional spectrometers. Overall performance is usually specified using the etendue-resolving power product (GR) and employed for the comparison of different spectrometers. For example, one can calculate using available datasheets that at the wavelength of $600 \mathrm{~nm}$ an Ocean Optics USB2000 spectrometer with a $25 \mu \mathrm{m} \times 1000 \mu \mathrm{m}$ slit, $\mathrm{NA}=0.22$, and $1200 \mathrm{~mm}^{-1}$ grating [17] has $\mathrm{GR} \approx 3.8 \mathrm{~mm}^{2} \mathrm{sr}$, while the Hamamatsu minispectrometer with a $70 \mu \mathrm{m} \times 550 \mu \mathrm{m}$ slit and $\mathrm{NA}=0.22$ [12] provides $\mathrm{GR} \approx 0.4 \mathrm{~mm}^{2} \mathrm{sr}$. The microspectrometer presented in this article has a smaller etendue-resolving power product of about $0.063 \mathrm{~mm}^{2} \mathrm{sr}$, which is due to the relatively small entrance slit and $\mathrm{NA}=0.14$. However, the performance of the device can be improved without sacrificing its small dimensions. The spectral resolution of the device can be increased in two ways. The pixel size of the photosensor should be reduced to provide at least two pixels for the registration of a monochromatic entrance slit image. At the same time, a diffraction grating with smaller pitch will contribute to the higher spectral resolution irrespec- tive of the photosensor. Numerical simulations demonstrate that it is possible to achieve a $0.4 \mathrm{~nm}$ resolution with a $6 \mu \mathrm{m} \times 400 \mu \mathrm{m}$ entrance slit and $\mathrm{NA}=0.22$, which results in a $0.54 \mathrm{~mm}^{2} \mathrm{sr}$ etendue-resolving power product. Increase in the diffraction angles results in a larger dimension of the device along the direction of the dispersion (direction oy in Fig. 1). However, this dimensional increase does not exceed $3-4 \mathrm{~mm}$, and the largest dimension of the spectrometer remains defined by the focal distance of the mirror. In addition to that, it is not practical to make the grating pitch smaller than about $1 \mu \mathrm{m}$, since the gain in the resolution due to the increased dispersion would be annihilated by the large off-axis aberrations of the mirror, which cannot be compensated by the grating design.

Future work is focused on the integration of the entrance slit, an aberration-correcting grating, and a customized linear array of long and narrow pixels on a single silicon chip. In mass production an array of spherical mirrors can be replicated on a plastic substrate, which can be subsequently aligned and bonded to the processed wafer containing spectrometer chips. When diced, such a bonded wafer yields a number of assembled spectrometers.

The authors acknowledge the financial support from the Dutch Technical Foundation (STW) under grant DET.6667. We are indebted to Lina Sarro and Charles de Boer (DIMES, TU Delft) for input on wafer processing and to Jan Groeneweg and Jan Cornelis Wolff (DIMES, TU Delft) for mask fabrication.

\section{References}

1. R. F. Wolffenbuttel, "MEMS-based optical mini- and microspectrometers for the visible and infrared spectral range," J. Micromech. Microeng. 15, S145-S152 (2005).

2. O. Manzardo, R. Michaely, F. Schädelin, W. Noell, T. Overstolz, N. De Rooij, and H. P. Herzig, "Miniature lamellar grating interferometer based on silicon technology," Opt. Lett. 29, 1437-1439 (2004).

3. S. Ura, F. Okayama, K. Shiroshita, K. Nishio, T. Sasaki, H. Nishihara, T. Yotsuya, M. Okano, and K. Satoh, "Planar reflection grating lens for compact spectroscopic imaging system," Appl. Opt. 42, 175-180 (2003).

4. S. Grabarnik, R. Wolffenbuttel, A. Emadi, M. Loktev, E. Sokolova, and G. Vdovin, "Planar double-grating microspectrometer," Opt. Express 15, 3581-3588 (2007).

5. I. Avrutsky, K. Chaganti, I. Salakhutdinov, and G. Auner, "Concept of a miniature optical spectrometer using integrated optical and micro-optical components," Appl. Opt. 45, 7811-7817 (2006).

6. J. H. Correia, G. de Graaf, S.-H. Kong, M. Bartek, and R. F. Wolffenbuttel, "Single-chip CMOS optical microinterferometer," Sens. Actuators A, Phys. 82, 191-197 (2000).

7. S.-W. Wang, C. Xia, X. Chen, W. Lu, M. Li, H. Wang, W. Zheng, and T. Zhang, "Concept of a high-resolution miniature spectrometer using an integrated filter array," Opt. Lett. 32, 632-634 (2007).

8. D. L. Kendall, W. P. Eaton, R. P. Manginell, and T. G. Digges III, "Micromirror arrays using KOH:H2O micromachining of silicon for lens templates, geodesic lenses, and other applications," Opt. Eng. 33, 3578-3588 (1994). 
9. J. Albero, L. Nieradko, C. Gorecki, H. Ottevaere, V. Gomez, J. Pietarinen, "Si moulds for glass and polymer microlenses replication," Proc. SPIE 6992, 69920A (2008).

10. S. Grabarnik, A. Emadi, E. Sokolova, G. Vdovin, and R. F. Wolffenbuttel, "Optimal implementation of a microspectrometer based on a single flat diffraction grating," Appl. Opt. 47, 2082-2090 (2008).

11. W. G. Fastie, "Small plane grating monochromator," J. Opt. Soc. Am. 42, 641-647 (1952).

12. http://jp.hamamatsu.com/products/sensor-ssd/pd186/4009/ C9409MA/index_en.html.
13. ZEMAX Optical Design Program, User's Guide, Version 9.0 (Focus Software, 2000).

14. C. Palmer and W. R. McKinney, "Imaging theory of planesymmetric varied line-space grating systems," Opt. Eng. 33, 820-829 (1994).

15. http://www.videologyinc.com.

16. K. Burns, K. B. Adams, and J. Longwell, "Interference measurements in the spectra of neon and natural mercury," J. Opt. Soc. Am. 40, 339-344 (1950).

17. http://oceanoptics.com/products/usb2000+.asp. 